\title{
ZUR GEOMORPHOLOGIE DES NIEDERSIMMENTALES
}

\author{
Von ERwin Genge
}

Mit 4 Abbildungen

\section{TOPOGRAPHISCHE ÜBERSICHT}

Das Simmental zerfällt geographisch und politisch ins Ober- und ins Niedersimmental. An das $\mathrm{S}-\mathrm{N}$ gerichtete obere Talstück schließt sich die $\mathrm{W}-\mathrm{E}$ orientierte untere Talhälfte. Durch diese Richtungsänderung entsteht nicht nur ein klimatischer Unterschied, sondern auch ein großer landschaftlicher Gegensatz. Aus dem Quertal ist ein Längstal geworden. Der einfacheren, kulissenartigen Geländeform der obern Gegend steht die reichgegliederte Terrassenlandschaft des untern Teilstückes gegenüber. Wenn wir uns im Nachstehenden im Niedersimmental hauptsächlich mit dieser Terrassenlandschaft beschäftigen, so deshalb, weil sie dort besonders stark in Erscheinung tritt.

Das untere Simmental (Abb.1) beginnt dort, wo die Simme aus der SW-NERichtung in die W-E-Richtung umbiegt, zirka $2 \mathrm{~km}$ SW Oberwil und etwa 1,5 km $\mathrm{N}$ der politischen Grenze zwischen Ober- und Niedersimmental entfernt, nahezu in der Mitte zwischen den beiden Ortschaften Boltigen und Oberwil. Die Biegungsstelle ist eine Talenge, im Volksmund kurz "Enge» genannt. Von hier an erstreckt sich das Längstal bis zur "Port», ebenfalls eine Talenge, $1 \mathrm{~km} \mathrm{SW} \mathrm{Wimmis} \mathrm{(Abb.} \mathrm{2).}$ Mit dem Austritt aus ihr endet geographisch das Niedersimmental, während es politisch bis an den Thunersee reicht.

Geologisch folgt das Niedersimmental der großen Mulde zwischen den Kalken und Schiefern der Stockhornkette und der Spillgertengruppe, die beide der Klippendecke angehören. Diese Mulde ist von Flysch ausgefüllt, der ein allgemeines W-E-Streichen besitzt und damit ziemlich genau der Talrichtung folgt. Im einzelnen zeigen sich aber kleinere Abweichungen im Streichen wie auch im Fallen, das um die Senkrechte herum schwankt. Bei Oberwil reicht der Flysch bis auf $1200 \mathrm{~m}$ Höhe hinauf, sinkt aber talauswärts bis Latterbach unter $800 \mathrm{~m}$. Um die Ursache der beiden Talverengerungen, der "Enge " im W und der "Port" im E, festzustellen, genügt ein Betrachten der geologischen Karte'. In der "Enge" überquert ein widerstandsfähiger Gesteinszug der Simmendecke im spitzen Winkel das Tal, so daß an dieser Stelle das eigentliche Flußbett der Simme durch die Felsen der Aptychenkalke auf eine Breite von kaum $5 \mathrm{~m}$ zusammengedrängt.ist. Es ist wohl hier das engste Flußbett des ganzen Tales. Den Querriegel der "Port" bildet dagegen der harte Malmkalk der Klippendecke. Das rund $17 \mathrm{~km}$ lange Teilstück zwischen beiden Talengen weist nur ein ausgesprochenes, breites Becken auf, das sich vom "Kastel", einem markanten Geländevorsprung SW Latterbach, bis zur "Port" ausdehnt.

Die Simme, früher auch Sibne oder kurzweg "Landwasser" geheißen, fließt ungefähr in der Mitte der langgestreckten Flyschzone durch das Niedersimmental. Größere Zuflüsse sind der Bunschibach bei Weißenburg und der etwas weiter talabwärts von rechts kommende Oeygrabenbach. Beide münden gleichsohlig in die Simme. Die Kirel ${ }^{2}$ aus dem Diemtigtal, der längste Zufluß, fließt bei Oey in den Hauptfluß. Das Hauptgewässer des Diemtigtales ist zwar der Filderich, der aber $4 \mathrm{~km}$ vor seiner Mündung in die Simme den Namen an die bedeutend kürzere Kirel verliert.

\section{DIE TERRASSENLANDSCHAFT DES NIEDERSIMMENTALES}

Der Flysch, in dem die Talsohle eingeschnitten ist, besteht zum Teil aus festen Sandsteinbänken, zum Teil aus bröckeligen Schieferschichten. Die dünnblättrigen Lagen verwittern leicht und verursachen oft Schlipfe. In diesem verhältnismäßig leicht

1 F. RÁbowskr: Simmental et Diemtigtal, 1:50000. Carte spéciale No 69, Mat. Carte géol. suisse, NS. 35.

2 Anmerkung: "Die» Kirel ist mehr im vordern Teil des Tales, "der" Kirel mehr im hintern Teil gebräuchlich. 
zerstörbaren Flysch bildete sich, wie schon erwähnt, im Gegensatz zum trogförmigen Obersimmental, eine ausgesprochene Terrassenlandschaft.

Auf den Terrassen bauten sich die Menschen auf der Sonnseite des Tales ihre Wohnstätten, hauptsächlich auf der untersten. Dort waren sie vor den früher öfters auftretenden großen Überschwemmungen der Simme sicher. Diese Terrassen gestatteten auch bequeme Verbindungswege zwischen den einzelnen Ortschaften. Die Staatsstraße zum Beispiel benützt sie von Latterbach bis Weißenburg.

Nach oben treten vornehmlich noch zwei Terrassensysteme im Gelände stärker hervor: ein tieferes in 1000 bis $900 \mathrm{~m}$ ü. M. und ein höheres in 1500 bis $1400 \mathrm{~m}$ ü. M. $\mathrm{Da}$ sie in der Höhenlage der benachbarten Burgfluh und Simmenfluh (Abb. 2) entsprechen, wurden sie von P. BECK ${ }^{3}$ als Burgfluh- und Simmenfluhniveau bezeichnet. Dagegen erhielt bisher die unterste Erosionsterrasse noch keinen Namen. Sie ist in Erlenbach gut ausgebildet und sei deshalb Erlenbachterrasse genannt ${ }^{4}$. Sie reicht von Außerlatterbach fast lückenlos bis Weißenburg und ist bis Oberwil feststellbar, und zwar beidseitig der Simme. Die Terrasse fällt ziemlich steil zum Flusse ab. Ihr oberer Rand liegt im allgemeinen 15-20 m über dem Wasserspiegel. In Oberwil beträgt ihre Meereshöhe $800 \mathrm{~m}$, in Erlenbach $700 \mathrm{~m}$ und kurz vor der Port noch $650 \mathrm{~m}$. Ihr Gefälle von 11,1\% stimmt beinahe mit dem der heutigen Simme (10,5\% überein. Diese Erosionsterrasse trägt durchwegs verschwemmien Gletscherschutt und nur in Außerlatterbach noch ursprüngliches Moränenmaterial.

Wo Seitenbäche in die Simme münden, ist die Erlenbachterrasse jeweilen stark erniedrigt, jedoch später wieder von Bachschutt überführt worden. Die Schuttkegel sind also den örtlich erniedrigten Terrassen aufgesetzt. Auf ihnen entstanden die ersten Siedlungen. Sie boten den Vorteil, das ganze Jahr aus dem Seitenbach genügend Wasser zur Verfügung zu haben und trotzdem vor Überschwemmungen der Simme geschützt zu sein. Bei heftigen Gewittern bestand jedoch die Gefahr eines seitlichen Ausbruches des Wildbaches. Das Andenken an solche Katastrophen lebt noch heute in Volkssagen fort. So soll zum Beispiel an Stelle des heutigen Heimwesens "Oberdorf" bei Erlenbach ein wirkliches Dorf gestanden haben.

Untersuchen wir die Hänge genauer, so finden wir außer den bereits erwähnten, im Gelände stark hervortretenden Terrassen noch weitere Reste von ehemaligen Talböden. Sie sind allerdings weder ausgedehnt noch zusammenhängend und bieten oft kaum Platz für eine kleine Heimstatt oder eine Scheune. Eine solche erste $Z$ wischenterrasse über der Erlenbachterrasse ist diejenige des "Kastel" bei Latterbach. Ihr entspricht die Stationsterrasse von Oberwil. Eine höhere Terrasse bildet unter anderm die Kuppe des Pfrundhubels von Erlenbach und der Boden des Dörfchens Bunschen bei Oberwil. Auf diesen beiden Zwischenterrassen wie auf den noch höher gelegenen liegt bis zum Burgfluhniveau hinauf vielfach Moränenmaterial, das im Gegensatz zum Flyschuntergrund der Hänge einen fruchtbaren Boden abgibt.

Auf Abb. 3, die als Beispiel für die Lage und Bodenbeschaffenheit eines Dorfes im Niedersimmental gelten mag, ist der Flyschsaum des Kleindorfschuttkegels zugleich der Steilrand der Terrasse von Erlenbach. Es wurde gerade dieses Dorf gewählt, weil nur von diesem eine sehr gute, neue Kartengrundlage existiert. Die Ausdehnung der Erlenbachterrasse in der untern Hälfte des Niedersimmentales ist auf Abb. 4 dargestellt.

Jeder Terrassenbildung entspricht eine Gefällsveränderung. Die Ursachen können entweder in einer Senkung des Vorlandes oder in einer Hebung des Hinterlandes oder in einer kombinierten Geländebewegung bestehen. Diese zahlreichen Erdkrustenbewegungen - entsprechend der Anzahl der Terrassen - fanden in der Zeit vor der größten Taleintiefung in den Alpen, in der Interglazialzeit $\mathrm{Ri} /$ Würm, statt; denn das Burgfluhniveau (1000-900 $\mathrm{m}$ ü. M. am Talausgang) stellt den Durchtalungszustand zu Beginn der Rißeiszeit dar, wie aus der Verbindung der Deckenschotter des Mittellandes mit diesem Niveau geschlossen werden darf ${ }^{5}$.

3 P. Beck: Grundzüge der Talbildung im Berner Oberland. Eclogae geologicae Helvetiae 16, 1921, S. 139-176.

- In einem unveröffentlichten Manuskript über Terrassenbildungen gebrauchte ich dafür den Ausdruck "Wilerterrasse", den dann P. KöCHLr in seiner Arbeit: "Die obere Grenze der Dauersiedlungen im Simmental in ihrer Abhängigkeit von Bodengestalt und Landwirtschaft ", Geographica Helvetica, II, 1947, S. 11, verwendete. Der Name "Erlenbachterrasse" ist in einer eigenen Studie veröffentlicht, betitelt: "Vom Boden und von der Besiedelung im Niedersimmental", 1944.

5 P. BECK: Zur Geologie und Klimatologie des schweizerischen Altpaläolithikums. Mitteilungen der Naturwissenschaftlichen Gesellschaft Thun, 4, 1939, S. 57-97. 


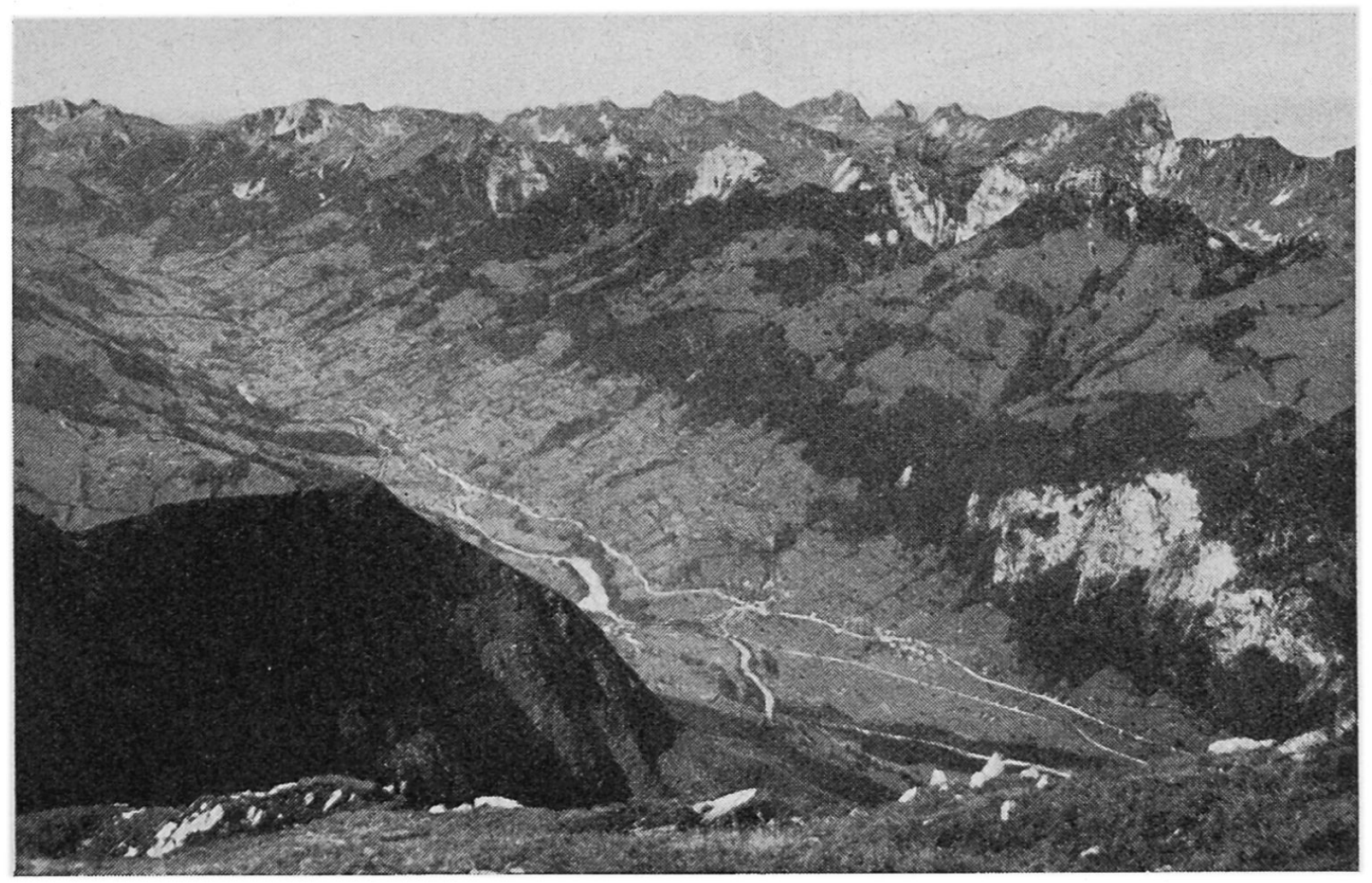

Abb. 1. Das Niedersimmental von Oberwil bis Außerlatterbach. Käufliche Ansichtskarte

\section{DAS SCHWEMMLAND DER SIMME}

Die Auterrasse. Blicken wir vom Steilrand der Erlenbachterrasse gegen die Simme hinunter, so bemerken wir etwa 12-15 m unterhalb des Terrassenrandes eine weitere Verflachung von 2 bis $3 \mathrm{~m}$ Höhe. Sie ist mit Unterbrechungen durch das ganze untere Simmental verfolgbar. Sie ist eine Akkumulationsterrasse, die auf Kies und Sand ausgebeutet wird. Ausnahmsweise ist sie auch in Flysch eingeschnitten. Wird die Simme, was sehr selten vorkommt, durch Murgänge von Seitenbächen gestaut, so werden diese Terrassen - nennen wir sie Auterrassen - überflutet (punktierte Flächen in Abb. 3 und Abb. 4). Deshalb finden sich auf ihnen keine älteren Behausungen, nur Ställe und Scheunen. Die Spiez-Zweisimmen-Bahn bedient sich ihrer bis wenig unterhalb Ringoldingen für ihre Geleiseführung.

Der jüngste Talboden. Dieser liegt 2-3 m tiefer als die Auterrasse, erhebt sich aber noch bis $80 \mathrm{~cm}$ über den normalen Simmewasserstand. Er trägt den buschigen Auwald und besteht nur aus Kies und Sand (weiße Fläche in Abb. 3 und 4). Er wird nur noch bei außerordentlichen Hochwasserständen überschwemmt.

Seitdem die Simme größtenteils korrigiert ist und Bahndämme auf dem jüngsten Talboden aufgeführt wurden (1897 Bahn bis Erlenbach, 1902 bis Zweisimmen), haben sich die Siedlungsverhältnisse in den Ebenen von Erlenbach und Latterbach teilweise geändert. Wohnhäuser und Ställe rücken nun gegen die Simme vor, und zugleich hat eine intensive Bearbeitung des früher wenig genutzten Talbodens eingesetzt.

Die erste Vorbedingung zu dieser Änderung lag in der Ableitung der Kander durch den Strättlighügel in den Thunersee in den Jahren 1712 bis 1714. Vorher hatte sich die Rückstauung der Simme durch die Kander bis nach Oey hinauf bemerkbar gemacht und das Auland in ungesundes Sumpfland verwandelt. Das Bewohnen des Aulandes hat seine Vor- und Nachteile. Ein Vorteil liegt im gesunden Grundwasser, das direkt beim Hause durch Sodbrunnen benützt werden kann. Früher machte man davon oft Gebrauch. Das höherstehende Grundwasser wirkt sich aber in diesem Boden für jede Grabung nachteilig aus. Auch dürfen die Keller, falls sie überhaupt möglich sind, nicht tief gegraben werden, sonst leiden sie stark unter Feuchtigkeit. 
Das Gand. Die vegetationslosen Sand- und Kiesbänke, die sich bis $50 \mathrm{~cm}$ über dem gewöhnlichen Wasserspiegel erheben, bezeichnen die Anwohner als "Gand", ohne zu ahnen, daß dieses Wort unverändert aus der Zeit stammt, da die Kelten diese Gegenden bewohnten. Die Bänke werden als Lieferanten von Șand und Kies geschätzt. Bei Hochwasser sind sie regelmäßig überflutet.

\section{DIE SIMME}

Sie fließt auf der $17 \mathrm{~km}$ langen Strecke Oberwil-Port nirgends über anstehenden Felsen. Er ist erst sichtbar am Ausgang der Port, direkt untenher des Stauwehres, wo sich die festen Bänke von Mytilusdogger quer durch das Simmebett ziehen. Infolge det rückwärtsschreitenden Erosion, die nach der Kanderablenkung (1714) einsetzte, wurde diese Schwelle bloßgelegt.

Im Staatsarchiv in Bern findet sich eine Notiz, wonach die Simme 1741 ein Stück Wiesland in der Größe von 5 Jucharten 19 Klaftern bei Wimmis weggerissen habe. Bei der heutigen Flußtiefe wäre dies nicht mehr möglich. Da die Tiefe des anstehenden Felsbettes auf dieser ganzen Strecke noch nie erbohrt oder festgestellt wurde, kann man über die Mächtigkeit der Schotterauffüllung nur Vermutungen hegen.

Über die Wasserführung der Simme, die im Hinblick auf ein kommendes Kraftwerk große Bedeutung erlangt hat, geben die folgenden Zahlen, die ich dem freundlichen Entgegenkommen der Baudirektion des Kantons Bern und der Bauabteilung der Bernischen Kraftwerke verdanke, guten Aufschluß. Alle Zahlenangaben bedeuten Kubikmeter pro Sekunde.

\begin{tabular}{lcccccc} 
Meßstationen & \multicolumn{2}{c}{$\begin{array}{c}\text { Durchschnittliche } \\
\text { maximale Jahresmenge }\end{array}$} & \multicolumn{2}{c}{$\begin{array}{c}\text { Durchschnittliche } \\
\text { minimale Jahresmenge }\end{array}$} & \multicolumn{2}{c}{$\begin{array}{c}\text { Durchschnittliche } \\
\text { mittlere Jahresmenge }\end{array}$} \\
& $1921-30$ & $1931-40$ & $1921-30$ & $1931-40$ & $1921-30$ & $1931-40$ \\
Oberwil & 14,90 & 14,40 & 7,43 & 8,90 & 11,80 & 11,90 \\
Oey-Diemtigen & 27,50 & 23,90 & 13,94 & 15,70 & 19,90 & 20,10
\end{tabular}

Wir ersehen daraus, daß die beiden zehnjährigen Mittelwerte jeweilen gut übereinstimmen. Die Schwankungen in der. Wasserführung sind beträchtlich, die durchschnittlichen Jahresabflußmengen jedoch während $20 \mathrm{Jahren}$ sehr ausgeglichen. Es dürften deshalb die gefundenen Resultate auch noch für eine längere Periode ihre Gültigkeit behalten. Der schwankende Wasserstand ergibt sich auch aus folgenden Zahlen:

Meßstationen

\begin{tabular}{|c|c|c|c|}
\hline \multicolumn{4}{|c|}{ Mittlere Tagesabflußmenge } \\
\hline & um & in & um \\
\hline $1921-30$ & $1931-40$ & $1921-30$ & $1931-40$ \\
\hline 93,8 & 70,7 & 1,69 & 1,53 \\
\hline 250,0 & 180,0 & 3,70 & 3,80 \\
\hline
\end{tabular}

Oberwil

Oey-Diemtigen

Die Differenz zwischen Normalstand und Hochwasserstand beträgt zirka $60 \mathrm{~cm}$, bei ganz außerordentlichem Hochstand auch schon $1,6 \mathrm{~m}$ (z. B. 14. 5. 1930). Das bedeutet, daß die mittlere Abflußmenge im Tag bis zu 70 Kubikmeter pro Sekunde geschwankt hat. Dieser Wechsel von Hoch- und Niederwasser bewirkt jeweilig das An- und Unterschneiden von Gehängen, das durch die Art des Materials (Flysch) noch gefördert wird.

\section{DIE VERFESTIGTEN SCHOTTER LÄNGS DER SIMME}

Über diese Vorkommen treffen wir in der geologischen Literatur nur spärliche Notizen. I. BACHMANN ${ }^{6}$ erwähnt die Nagelfluh von Latterbach und Oey. V. GilliÉRON $^{7}$ kennt ebenfalls diejenige von Oey, ohne näher darauf einzutreten. In der neueren Literatur kann einzig die geologische Karte Thun-Stockhorn ${ }^{8}$ angeführt werden. 1870.

- I. Bachmann: Die Kander im Berner Oberland. Ein ehemaliges Fluß- und Gletschergebiet. Bern

${ }^{7}$ V. Grllréron: Description géologique des Territoires de Vaud, Fribourg et Berne. Mat. Carte géol. suisse, XVIIIe livraison, 1885.

${ }^{8}$ P. BeCK und Ed. Gerber: Geologische Karte Thun-Stockhorn, 1:25000. Spezialkarte Nr. 96, Beitrag zur geologischen Karte der Schweiz, 1925. 


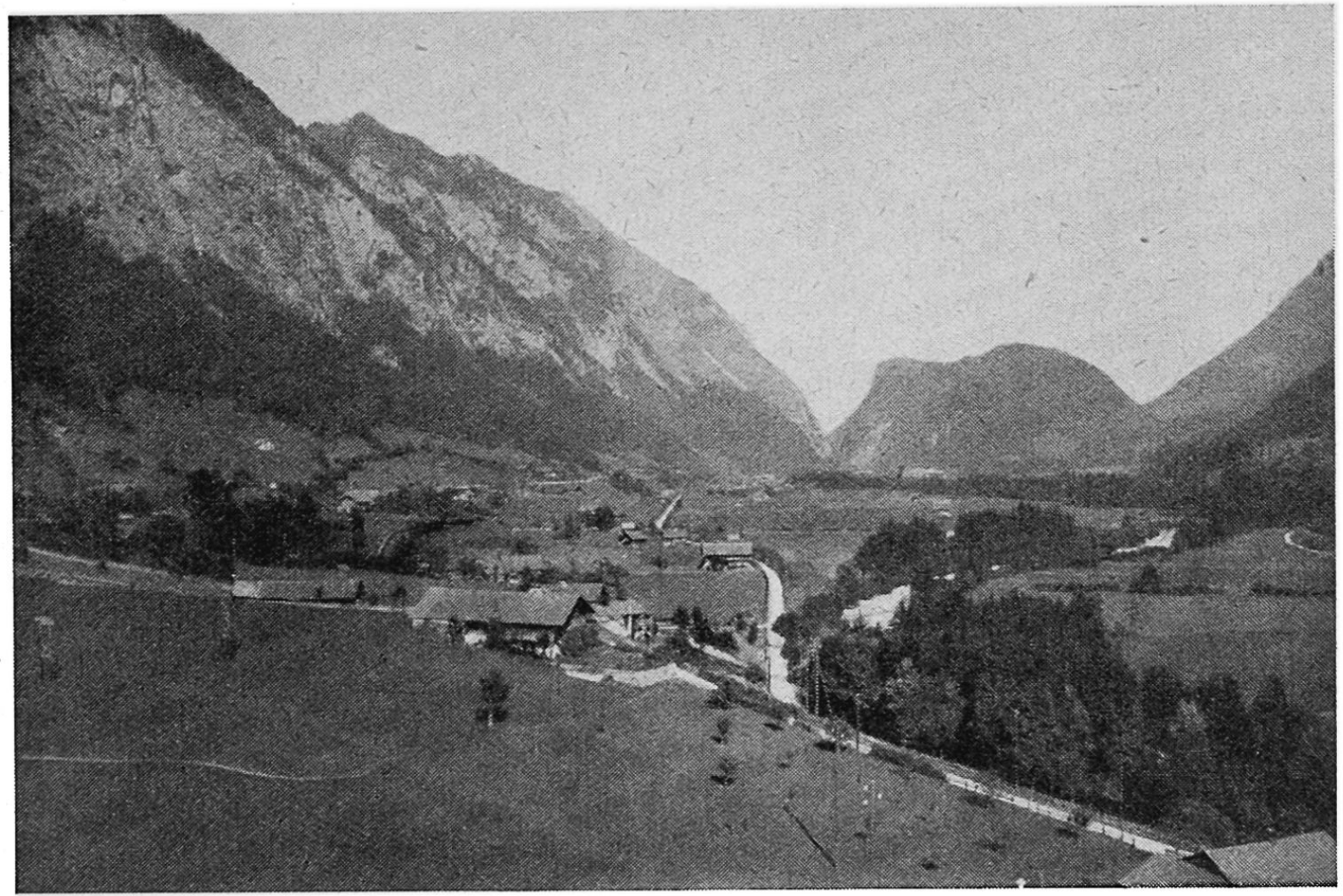

Abb. 2. Der Abschluß des Simmentals aus SW. Simmenfluh-Port-Burgfluh-Niesenfuß Photo R. Wenger, Erlenbach i. S.

Sie weist folgende Vorkommen auf (Numerierung nach Abb. 4): Ringoldingen (Metschenmatte, 3), Steinischuttkegel bei Erlenbach (4), «im Schlatt» (5), beim "Kastel» (6), Latterbach ("auf der Nagelfluh», bis «im Drittel», 9), Station Oey (7), in der Port (11). Dazu finden sich noch die weitern Vorkommen: Ringoldingen (Zelg, 1), Mühleboden (2), Erlenbach (Kleindorf, ohne Nummer), 200 m S Oey (linkes Kirelufer, ohne Nummer), E Oeyfeld (8) und S Burgholz (10). Bei Zelg (1) bestehen die verfestigten Schotter zum Teil aus groben, bis $1 \mathrm{~m}^{3}$ großen Blöcken. Sie verraten uns die damalige Gletschernähe. Die Partie «im Schlatt» (5) wurde bei einer Wegverbreiterung im Winter 1922/23 zum größten Teil, in der Port (11) aus dem gleichen Grunde im Jahre 1939 vollständig weggesprengt.

Die Meereshöhe des obersten Vorkommens (1) beträgt $703 \mathrm{~m}$, diejenige des untersten (11) $633 \mathrm{~m}$. Das Gefälle auf der $9 \mathrm{~km}$ langen Strecke beträgt $10 \%$, entspricht also demjenigen der Simme. Die ganze Schotterzone Zelg-Port hing wohl ursprünglich zusammen. Weiter flußaufwärts treffen wir erst nach $10 \mathrm{~km}$, in der Nähe von Boltigen, wieder Nagelfluh an.

Alle Vorkommen besitzen gemeinsame Merkmale. Sie haften am untern oder mittleren Teil des Steilrandes der Erlenbachterrasse direkt auf dem Flysch, das Vorkommen in der Port auf dem Malm. Ihre Mächtigkeit ist gering, höchstens einige Meter. Sie bilden senkrechte Wände oder Fluhabsätze von 1 bis $4 \mathrm{~m}$ Höhe, sind sehr fest verkittet und weisen oft Hohlkehlen auf, in denen die Anwohner etwa Gerätschaften oder Holz aufbewahren. Die Schichtung ist waagrecht und fällt teilweise leicht in den Berg, weist aber in Latterbach (9) und S Oey (linkes Kirelufer) im unteren Teil auch Deltastruktur auf, was auf einen ehemaligen See im Becken von Oey schließen läßt. Das Material besteht aus Gesteinen des Simmentales. Die Nagelfluh wird von losen, horizontalen Schottern aus verschwemmtem Gletscherschutt überlagert.

Zwischen den Vorkommen bei Oey ( 7 und 8) und zwischen 9 und 11 auf der gegenüberliegenden Seite liegen noch mittelhart verfestigte Schotter auf der Erlenbachterrasse, die gleichmäßigere Korn- 
größen aufweisen, weder Hohlkehlen noch hervortretende Bänke bilden und den Eindruck eiper verhältnismäßig frischen Ablagerung erwecken, wenn sie auch weniger frisch sind als diejenigen, die den jüngsten Talboden bilden.

$\mathrm{Da}$ die verfestigten Schotter direkt auf Flysch auflagern und von Moränenmaterial bedeckt sind, dürfen wir sie in die Interglazialzeit Riß/Würm stellen, und zwar vor Beginn der Würmeiszeit, da der Simmegletscher das Becken von Oey noch nicht erreicht hatte (Eiszunge oberhalb Ringoldingen). Der Kandergletscher foß vor der Port vorbei, so daß er die Schmelzwasser des Simmegletschers staute, die damit ihre. Strömungsgeschwindigkeiten verloren und die Kiesmassen ablagerten. Diese später verfestigten Schotter sind also Stauschotter. Die mittelhart verfestigten Schotter auf der Erlenbachterrasse stammen jedoch aus einer späteren Interglazialzeit (Würm I/Würm II ?) und die jüngsten Talschotter aus der Nacheiszeit.

Es ist nicht ausgeschlossen, daß unsere verfestigten Schotter im Zusammenhang mit ähnlichen Bildungen stehen, die zwischen Spiez, Thun und Bern zu beobachten sind ${ }^{9}$.

\section{DIE NAGELFLUHBILDUNGEN IM DIEMTIGTAL}

Auch das Diemtigtal besitzt seine verfestigten Schotter. Wandern wir längs der Kirel von Oey talaufwärts, so erblicken wir bald links, bald rechts des Tales Steilwände, bestehend aus löcheriger Nagelfluh, die das gleiche Aussehen besitzt wie diejenige des Haupttales. Setzen sich die Vorkommen auf der rechten Talseite aus kleinen, übereinandergelagerten Fluhabsätzen zusammen, so zeigt die linke Talseite bis $80 \mathrm{~m}$ hohe, senkrecht abfallende Wände, ein Beweis für die überaus feste Verkittung. Die Längenausdehnung beträgt vom Horboden bis $600 \mathrm{~m} \mathrm{~S}$ Oey $3 \mathrm{~km}$ (Abb. 4). Auffallend ist die Gesamtmächtigkeit von beinahe $120 \mathrm{~m}$. Die Schotter steigen von $710 \mathrm{~m}$ ü. M. auf rund $875 \mathrm{~m}$ (Diemtigen und Bächlen). Sie liegen direkt auf Triasdolomit und Rauhwacke. Im Horboden beginnen die Ablagerungen ebenfalls mit zum Teil recht großen Blöcken, die auch hier auf Gletschernähe zur Zeit ihrer Entstehung hinweisen. Die sie überlagernden Moränen sind nach ihrer Höhenlage in das Würm I (Gurtenstadium) zu setzen, so daß die Bildung dieser Schotter ebenfalls der Interglazialzeit $\mathrm{Riß/Würm} \mathrm{zuzuweisen} \mathrm{ist.}$

Auch hier hat offenbar eine, wenn auch gewaltigere Stauung, die mindestens eine Höhe von $880 \mathrm{~m}$ ü. M. erreichte, die Ablagerungen verursacht. Als Grund dieser Stauung fallen verschiedene Möglichkeiten in Betracht. Da der Simmegletscher sich jeweils früher aus dem Becken von Oey zurückzog, kann eine Stauung durch sein aktives Eis nicht in Erwägung gezogen werden. Am wahrscheinlichsten ist eine Stauung durch den quer vor der Port vorbeiziehenden Kandergletscher. Eine allseits befriedigende Erklärung besitzen wir aber noch nicht.

Eine kurze Erwähnung verdient noch die Umgebung der Wattfluh. Dort bestand schon vor der letzten Vergletscherung ein altes Kirelbett. Es wurde aber von Schottern (der späteren Nagelfluh) aufgefüllt. Nach dem endgültigen Rückzug des Kirelgletschers fand der Bach seinen alten Lauf nicht mehr und schnitt sich ein neues Bett in die Kalke der Trias. Es ist die enge Stelle am Fuße der Wattfluh, durch die heute auch die Straße führt.

\section{VON DEN EISZEITEN IM NIEDERSIMMENTAL}

Vom eiszeitlichen Simmegletscher ist nur mehr der Rätzligletscher zuhinterst in der Lenk, dessen Zunge auf $2280 \mathrm{~m}$ ü. M. hinunterreicht, übriggeblieben. Von den beiden ersten Eiszeiten (Günz und Mindel) sind keine Ablagerungen festzustellen. Die in den Bergen besonders stark arbeitende Verwitterung hat seither viele Geländeformeñ verändert und etwaigen Gletscherschutt weggeräumt. Zudem bestand der heutige Talboden am Ende dieser Eiszeiten noch gar nicht. Er lag damals ungefähr auf der Höhe der Wimmiser Burgfluh, des Diemtigbergli und des Roßberges bei Oberwil (Burgfluhniveau). Alle Gletscherablagerungen unterhalb dieser Höhe sind jünger als $\mathrm{RiB}$.

- Ed. Gerber: Uber ältere Aaretalschotter zwischen Spiez und Bern. Mitteilungen der Naturforschenden Gesellschaft Bern, 1914, S. 1-39. 


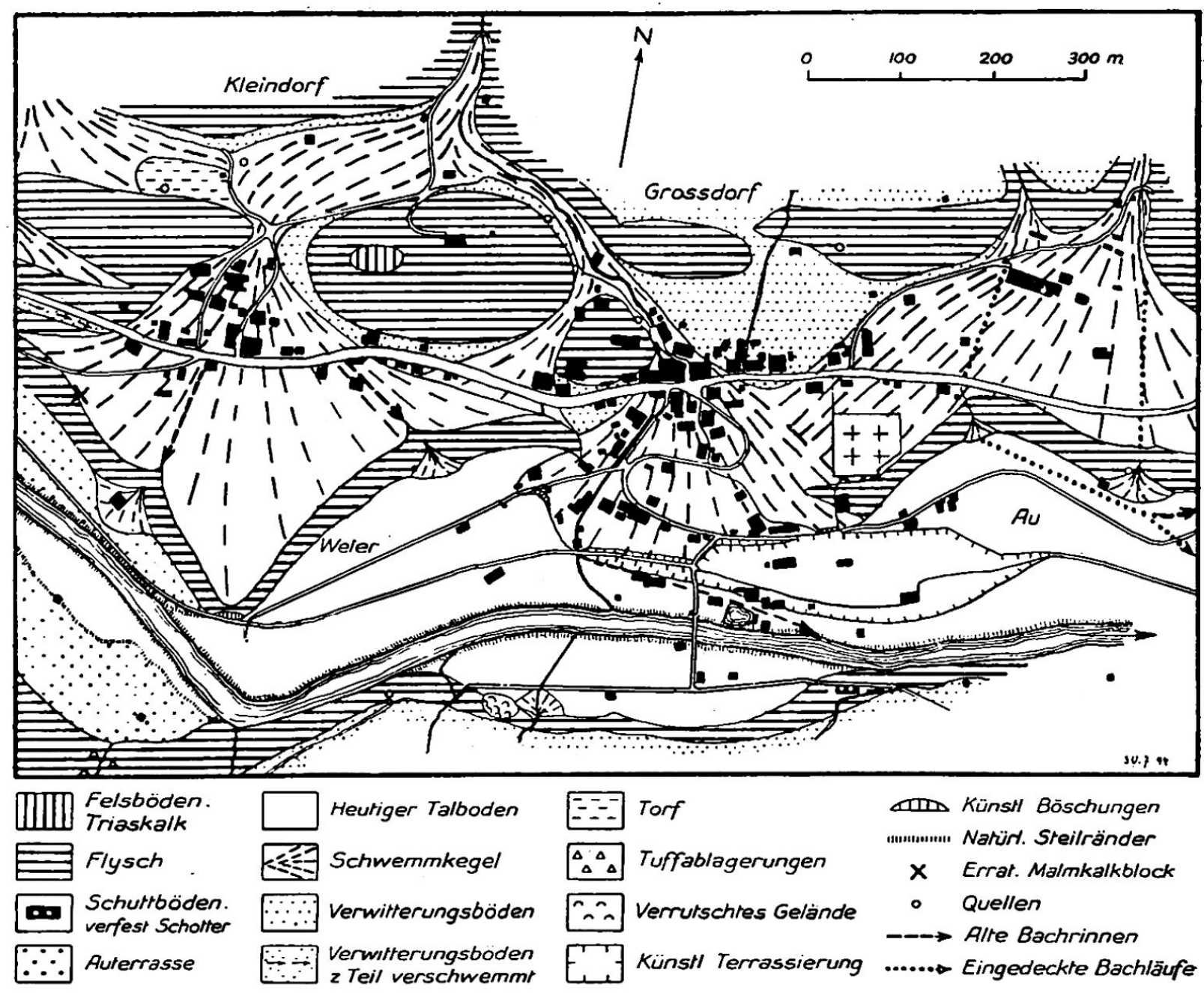

Abb. 3. Geologische Karte von Erlenbach

Die Rißvereisung machte sich zuerst durch den vorrückenden Kandergletscher bemerkbar, der einen Seitenarm bis ins Becken von Oey sandte, wie aus einem Block Gasterngranit geschlossen werden konnte, den man vor 70 Jahren am SW-Fuß der Burgfluh gefunden hat. Später stießen die vereinigten Diemtigtalgletscher von Kirel und Filderich in diese Gegend vor. Zuletzt erschien dort auch der Simmegletscher, weil er den weitesten Weg von seinem Nährgebiet bis hieher zurückzulegen hatte ${ }^{10}$. Durch das ständige Nachfließen des Simmeeises stieg dieses über den Eisarm des Kandergletschers hinauf und floß hoch über der Port ab, wie Gletscherschliffe in $1400 \mathrm{~m}$ Höhe an der Simmenfluh beweisen. Bei Weißenburg erreichte der Höchststand $1540 \mathrm{~m}$, ob der oberen Hausallmend (zwischen Stocken- und Mieschfluh) $1620 \mathrm{~m}$ und beim Pfrundnacki ob Allmenden finden sich die höchsten Rißmoränen in $1600 \mathrm{~m}$ ü. M. ${ }^{11}$. Diese auffallende Höhenlage ist auf die Stauung des Simmegletschers durch den Kandergletscher und die Burgfluh zurückzuführen. Bei diesem maximalen Eisstand wurden auch der Heitiberg und die benachbarte Simmenfluh überschwemmt. Die Eisoberfläche zog $150 \mathrm{~m}$ höher darüber hinweg, um dann in einem Gletscherbruch in der Richtung gegen Reutigen auf den Kandergletscher zu stürzen.

10 P. BecK: Eine Karte der letzten Vergletscherung der Schweizer Alpen. 1. Mitteilung der Naturwissenschaftlichen Gesellschaft Thun. 1926, S. 3-53.

11 P. BeCK und Ed. Gerber: Geologische Karte Thun-Stockhorn, 1:25000. Geologische Spezialkarte Nr. 96, Beitrag zur geologischen Karte der Schweiz, 1925. 
Dieser höchste Eisstand dauerte verhältnismäßig wenig lange. Die Gletscheroberfläche senkte sich um rund $50 \mathrm{~m}$ und lagerte dann wieder Seitenmoränen ab. Am Ende der Rißeiszeit zog sich der Simmegletscher als erster aus dem Becken von Oey zurück. Seine Schmelzwasser bildeten, weil der Ausgang durch die Port vom Diemtigtal- und Kandereis noch versperrt war, einen Stausee, in dem sich die später verfestigten Schotter der Simme ablagerten. Vielleicht ist auch die Bildung von Seeletten in der Port in jene zwischeneiszeitliche Periode zu setzen. Diese Lehme kamen anläßlich der Eröffnung eines Steinbruches, der zur Gewinnung von Malmkalk $900 \mathrm{~m}$ südwestlich der Wimmisbrücke angelegt wurde, erstmals im Herbst 1942 zum Vorschein. Die Seeletten, die direkt am polierten Malm anliegen, sind durch den fortschreitenden Steinbruchbetrieb fast gänzlich entfernt worden. Ihre Ausdehnung betrug $100 \mathrm{~m}$ in der Länge und $12 \mathrm{~m}$ in der Breite.

Diesen Ablagerungen der Interglazialzeit Riß/Würm war eine Erosion vorausgegangen, die das Simmental bis auf den heutigen Talgrund eingetieft hatte.

Während der beginnenden Würmeiszeit wiederholten sich die geschilderten Vorgänge im Becken von Oey, nur erreichten die Eismassen nicht mehr ihre frühere Mächtigkeit und Ausdehnung. Die Würmmoränen liegen einige hundert Meter tiefer. Diejenigen aus der ersten Hälfte der Würmeiszeit, des Würm I oder Gu r tens ta diu ms, liegen auf den Zwischenterrassen zwischen Burgfluhniveau und Erlenbachterrasse. In der darauffolgenden kurzen Interglazialzejt, der Spiezer Schwankung, verschwemmten die Schmelzwasser die Grundmoränen und breiteten sie auf der Erlenbachterrasse aus. Das Bernstadium (Würm II), die zweite Hälfte der Würmeiszeit, sah den Simmegletscher nur mehr bis Weißenburg vorrücken. Die untere Talhälfte blieb eisfrei12, während der Diemtigtalgletscher noch zum Simmental hinausreichte. Als im Bühlstadium der Kirelgletscher allein noch mit seiner Zunge bei Oey lag, war auch schon der unterste Teil des Obersimmentales bis Garstatt vom Eise entblößt ${ }^{13}$. Nun konnte sich der jüngste Talbodenschotter ablagern, und die Erlenbachterrassen erhielten ihre Schuttkegel aufgesetzt. In einer spätern Erosionsphase schnitt sich die Simme wieder ein; diesmal aber in ihre eigenen Ablagerungen. Es entstanden die Auterrassen. Ḋ̀r Vorgang kam aber zum Stillstand, bevor der Talfluß sein ehemaliges tiefstes Felsbett erreicht hatte. Heute kann die Simme, mit Ausnahme von Hochwasserzeiten, nur mehr akkumulieren, und die Schuttkegel werden weder weiter aufgeschüttet noch erodiert. Sie sind abgestorben. Ähnliche Beobachtungen machte auch F. Nussbaum im Saanegebiet ${ }^{14}$.

Am Schlusse sei doch der alten Sage aus dem Diemtigtal gedacht, die uns D. GempeLER ${ }^{15}$ überliefert hat. Ahasver kam auf seinen Wanderungen auch nach Schwenden im Diemtigtal. Das erstemal traf er die ganze Gegend vergletschert an. Als er zum zweitenmal dorthin kam, glänzte ihm ein See entgegen, und später befand er sich dort in einer sonnigen, südlichen Gegend, die mit Weinreben bepflanzt war. Bei seiner vierten Wiederkehr bot sich ihm das Bild von heute, eine grüne Alpenlandschaft. Einem Sennen, der ihm begegnete, teilte er mit, daß bei seinem nächsten Besuch das Tal wiederum vom Gletscher erfüllt sein werde. Das Bemerkenswerte an dieser Sage ist, daß die Menschen den Wechsel eines Klimas im Laufe der Jahrtausende erkannt haben. Erstaunlich ist, wie die Reihenfolge der Veränderungen der Landschaft in der Sage mit den tatsächlich erfolgten übereinstimmt. Auch im Volke lebt also die Vorstellung einer ehemaligen großen Vereisung. Der Zeitabschnitt der Seebildung ist als die wasserreiche Zeit beim Zurückschmelzen der Gletscher zu erklären. Damals geschah es oft, daß nach dem Eisfreiwerden der Talseiten die Hänge ins Rutschen kamen, da der Seitendruck des Eises wegfiel. Dadurch bildeten sich in der Talsohle zeitlich begrenzte Stauseen, die dann wieder verschwanden. Wir kennen auch eine viel wärmere Zeit als heute, in der die Gletscher eine bedeutend geringere Ausdehnung besaßen. Dieses günstige Klima herrschte vor ungefähr 4000 Jahren. (Eichenmischwald, $5000-3500$ v. Chr.) ${ }^{18}$

12 P. Beck: Studien über das Quartärklima im Lichte astronomischer Berechnung. Eclog. geol. Helvetiae, 30, 1937, S. 75-86, und 31; 1938, S. 137-172.

13 F. Nussbaum: Die eiszeitliche Vergletscherung des Saanegebietes. Jahresbericht der Geographischen Gesellschaft Bern, 29, 1906, S. 1-230.

14 F. Nussbaum: Die Täler der Schweizer Alpen. Bern 1910, S. 108.

15 D. Gempeler: Sagen und Geschichtliches aus dem Simmental, 5. Bd. Thun 1912, S. 91.

$16 \mathrm{M}$. WELTEN: Pollenanalytische, stratigraphische und geochronologische Untersuchungen aus dem Faulenseemoos bei Spiez. Zürich 1944. 


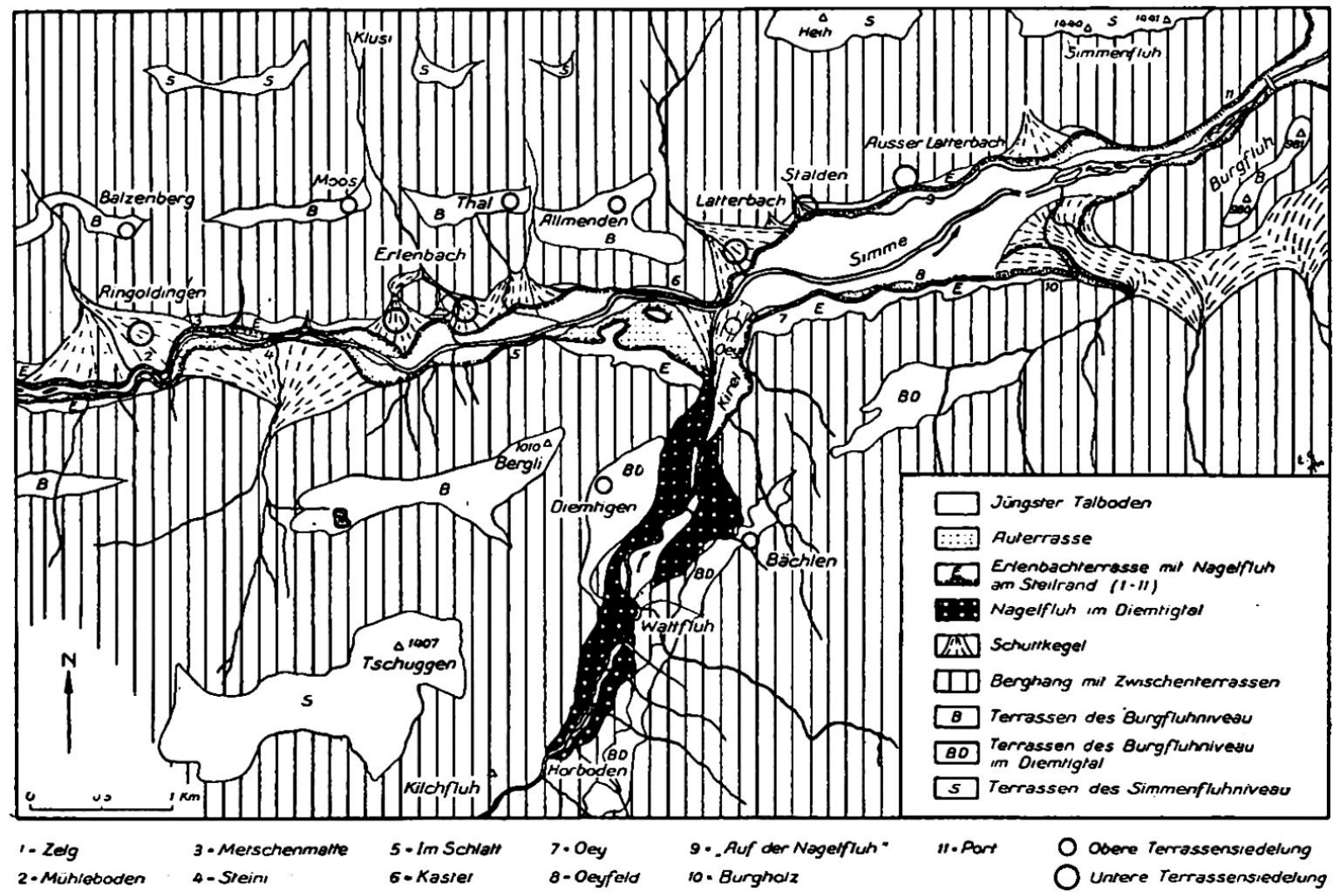

Abb. 4. Die hauptsächlichsten Terrassen und der verfestigte Schotter des Niedersimmentals. Die Terrassen des Burgfluhniveaus im Diemtigtal (BD) sind gegenüber denjenigen des Haupttales infolge späterer erneuter Erosion etwas niedriger

Die Prophezeiung einer neuerlichen Vereisung unserer Täler stellt die Antwort dar auf die etwa geäußerte Frage: Ist die Eiszeit endgültig vorbei oder leben wir in einer $Z$ wischeneiszeit? Die Sage bejaht die zweite Frage, während wir uns mit der Erkenntnis begnügen müssen: Wir wissen es nicht.

\section{DIE BODENFORMEN OBERHALB DES BURGFLUHNIVEAUS}

Der Vergletscherung verdanken wir die fruchtbare Erde auf den Terrassen: den verschwemmten Gletscherschutt auf der Erlenbachterrasse, die Moränen aber auf allen übrigen Terrassen bis zum Burgfluhniveau. An ihm haben teil die Terrassen vom obern Weißenburgberg $(1085 \mathrm{~m})$, von Grubi $(1060 \mathrm{~m})$, Balzenbergallmend $(1050 \mathrm{~m})$, Oberberg $(990 \mathrm{~m})$ und Thal-Allmenden auf der nördlichen, Roßberg $(1130 \mathrm{~m})$, Pfrundweid $(1058 \mathrm{~m})$ und Diemtigbergli $(1000 \mathrm{~m})$ auf der südlichen Talseite, um dann in der Mitte des Talausganges in der Port mit der Burgfluh (981 m) selber zu enden.

Die Höhe dieser Niveaus bedeutet auch den Abschluß der untersten Flyschzone mit ihren kontinuierlichen Terrassenbildungen. Auf der linken Talseite folgt nach kurzen, steilen Schutthängen eine erste, kleine Felsstufe von 50-150 m Höhe, gebildet aus den steilgestellten Malmschichten der Klippendecke. Dahinter schließt sich eine flachere, stellenweise sogar muldenförmige Zone an. Die Mulden sind kleine Combes. Die Gefällsveränderung ist durch die Formation des Mytilusdoggers bedingt, der sich an den Malm anschmiegt und infolge seiner Kohlenführung während der Kriegsjahre 1940-1945 neuerdings zu Bedeutung gelangte. Obwohl sich die Kohlen von Oberwil bis Allmenden vorfinden ${ }^{17}$, wurden sie nur ob Erlenbach (Bergwerk Klusi) in größerem Maßstabe abgebaut. Wenn auch die Kohle nicht erstklassig war, so half sie doch eine Notlage überbrücken und brachte Verdienst ins Tal.

17 E. Genge: Ưber die Kohlenvorkommen im Ostende der Gastlosenschuppe. Eclog. geol. Helvetiae, 35, 1942. S. 293-311. 
Nach steilen, bewaldeten Hängen stellt sich eine zweite Felsstufe ein, ebenfalls aus Malm, die aber eine Höhe von 300 bis $400 \mathrm{~m}$ aufweist. Es sind die Flühe vom Loherenspitz, die Stocken-, Miesch-, Walpersberg- und Brämenfluh sowie der Nüschletengrat. Ihre Höhen wechseln zwischen 1850 und $1950 \mathrm{~m}$ ü. M.

Zwischen diesen beiden Felsstufen befinden sich auf zum Teil flachen Böden ausgedehnte Alpweiden. Sie gehören ins Simmenfluhniveau und breiten sich in der Höhe dieser Fluh $(1460 \mathrm{~m})$ über das "Heiti» $(1492 \mathrm{~m})$ aus. Die beiden Stockenseen gehören ebenfalls diesem Niveau an. Sein Boden besteht aus dem Verwitterungsprodukt des darunterliegenden Felsens und eignet sich deshalb nur noch als Weideland.

Die rechte Talseite nimmt in ihrem Aufbau nicht genau denselben Verlauf, trotzdem sie zur Hauptsache auch der Klippendecke angehört. Sie wird aber hier zum Teil noch durch eine andere Decke, die Simmendecke, überlagert, die sich westlich des Roßberges bei Oberwil bis nahe ans westliche Ufer der Kirel bei Oey erstreckt. Eine erste Malmstufe fehlt, doch sind dafür die Hänge, infolge des widerstandsfähigeren Gesteins der Simmendecke, als Schutthänge steiler als die gegenüberliegenden. Das Simmenfluhniveau ist nur durch die Tschuggenalp $(1400-1500 \mathrm{~m})$ vertreten. Daß zwischen dem Burgfluhund Simmenfluhniveau noch weitere Terrassen existieren, beweisen diejenigen von Klusialp und die gegenüberliegende Feldmöseralp (Erlenbach) sowie der Lamperenhubel ob Oberwil.

Der Malm, der die beiden linksseitigen, typischen Felsstufen bildet, gibt auch den höhergelegenen Bergformen sein Gepräge. Er ist die dominierende Schicht, der sich die übrigen Ablagerungen, die hangende Obere und Untere Kreide und der liegende Dogger und Lias, anpassen. In $1800 \mathrm{~m}$ ü. M. haben wir nach P. BECK ${ }^{18}$ weitere Überreste eines Talbodens und auf $2000 \mathrm{~m}$ ü. M. ein ausgesprochenes Grat- und Gipfelplateau (Stüßligrat, Lasenberg und Walpersbergli). Nach oben schließen die Berge ab mit den Felshäuptern in $2100-2200 \mathrm{~m}$ ü. M. (Stockhorn, Hohmaad, Nünenen, Gantrisch, Bürglen und Ochsen) auf der linken, Grathörnli, Seefluh und Geißfluh auf der gegenüberliegenden Talseite.

Die Untersuchungsergebnisse vermitteln folgendes Gesamtbild:

\begin{tabular}{|c|c|c|c|c|}
\hline Hauptterrassen & $\begin{array}{l}\text { Höhe } m \text { ü. } M \\
\text { W } E\end{array}$ & Bodenbeschaffenheit & Besiedlung & Verbindung \\
\hline Simmenfluhniveau & $1600-1400$ & Magerer Verwitterungsboden & Sennhütten & Saum- und Fußwege \\
\hline Burgfluhniveau & $1100-900$ & Fruchtbarer Moränenschutt & Weiler & Wege \\
\hline Erlenbachterrasse & $.800-650$ & Fruchtbarer, fluvioglazialer & Dörfer & Straßen, Eisenbahnen \\
\hline
\end{tabular}
Schutt

Ein Überblick über die Tabelle zeigt, daß die Besiedlungsform des Tales, die Alpwirtschaft auf den Höhen und beschränkter Ackerbau im Talgrund die beste Ausnützung der von der Natur gegebenen Verhältnisse darstellten.

\section{AU SUJET DE LA GEOMORPHOLOGIE DU BAS-SIMMENTAL}

La vallée de la Simmen est creusée dans le synclinal séparant les anticlinaux du Stockhorn et des Spillgerten. Cette dépression est occupée par des grès et marnes du Flysch ainsi que des roches de la Nappe de la Simmen. Dans le Bas-Simmental se rencontre au niveau de la Burgfluh le niveau d'un talweg $(900$ à $1000 \mathrm{~m}$.). Au-dessus de la vallée s'étend une autre terrasse plus petite et plus basse (Erlenbach 650 à $800 \mathrm{~m}$.). Le long de son bord, on observe des poudingues antérieurs au Würm, à peu près du même âge que les graviers consolidés du Diemtigtal. Les dépôts glaciaires datent du Riss et du Würm.

\section{SULLA GEOMORFOLOGIA DELLA VALLE INFERIORE DELIA SIMME}

La valle inferiore della Simme, incisa nel Flysch, rappresenta un paesaggio a terrazze. Si distinguano tre livelli dominanti: a) la terrazza della Simmenfluh $(1600-1400 \mathrm{~m}$.); b) la terrazza della Burgfluh $(1100-900 \mathrm{~m}$.$) ; c) la terrazza di Erlenbach (800-650 \mathrm{~m}$.). Agli orli di quest'ultima affiorano le puddinghe, depositatè nell'epoca glaciale del "Praewürm v. Alla stessa epoca risalgono le puddinghe della parte inferiore di Diemtigen. I depositi glaciali datano dal Riss al Würm.

18 Siehe Anmerkung 2. 